\title{
Considerations on design, development and testing of Electrical Machines for automotive HVAC
}

\author{
Radu Martis ${ }^{1, *}$, Florin Pop-Piglesan ${ }^{1}$, Sorin Cosman $^{1}$, and Claudia Martis ${ }^{1}$ \\ ${ }^{1}$ Technical University of Cluj-Napoca, 400114 Memorandumului 28, Cluj-Napoca, Romania
}

\begin{abstract}
HVAC represents one of the highest energy consumer in a vehicle and for full electric vehicles, the design of HVAC and the dimensioning of its driving system is of utmost importance in order to avoid the limitation of driving range. Due to its advantages, especially when it comes to power density, PMSM is one of the most used electrical machines for a wide range of automotive applications, including HVAC systems. The paper presents the generation of the requirements, design, analysis and HiL testing of a PMSM for HVAC applications. The challenge is to develop a machine answering to the requirements for an electric vehicle HVAC at lowvoltage. An experimental model of the machine was tested using a testbench, based on HiL techniques.
\end{abstract}

\section{Introduction}

The automotive sector is in constant need of newer, cheaper and more efficient electrified components. This is a vast field, comprised of various applications, such as propulsion, steering, driving assistance, Heating, Ventilation and Air Conditioning (HVAC), windows lift, etc.

Among the automotive auxiliaries, HVAC systems represent the highest energy consumer in a vehicle. This is even more critical when it comes to electric vehicle [1]. The combination of high temperature and high relative humidity serves to reduce thermal comfort and indoor air quality [2]. From a state of thermal neutrality or comfort, any change will be perceived as unpleasant [3]. Thermal comfort research models take into account flexibility in indoor thermal conditions that extend the range of temperatures considered comfortable and incorporate strategies such as natural ventilation [5].

The need for higher comfort and luxury within a vehicle, either automobile, bus or commercial vehicles, in the context of transport electrification, is driving the demand for the development of eco-friendly high performing HVAC systems [6, 7]. The challenge is the proper selection of compressor and electric drive, in order to achieve accurate temperature and humidity control, better power supply quality, energy savings, less noise, higher safety and greater comfort $[8,9]$.

\footnotetext{
* Corresponding author: martis.radu@gmail.com
} 
In a fully electrical vehicle (FEV), where the available energy is the electricity stored in the battery pack, the HVAC system has an important impact on the driving range, that can be considerably decreased depending on the size of the HVAC and the operation scenario of the vehicle $[10,11,12]$. Besides that, automakers are asking for customized and modular solutions that allow the installation in conventional and unconventional locations in the vehicle, posing serious challenges to HVAC producers in terms of integration level and energy efficiency.

The automotive HVAC system operates under variable loads as it has to provide driver and passengers' comfort for a wide range of outdoor temperature and humidity conditions. Therefore, a careful design and proper operation strategies are necessary to achieve a reasonable autonomy of the vehicle without compromising the thermal comfort $[12,13]$.

The core of the modern automotive HVAC systems is built around a variable speed compressor. The electric driven compressor has several advantages: low emissions, flexible packing, efficient variable speed operation. However, it has several design challenges: low voltage level, robust construction, installation volume limitation, tough environmental conditions to withstand. Over the years, many types of motors have been used in this type of application. In the early years of development, the Split Phase Motors (SPM) were the most used, however, Permanent Magnet Synchronous Machines (PMSM) have gained ground. PM-less machines such as the Switched Reluctance Machine (SRM) or the Synchronous Reluctance Machine (SynRM), are considered as possible alternatives [14, 15]. They have proved to have good performances due to the advancement in materials being used, as well as more advanced and more reliable and performant control strategies, as well as semiconductor materials and power electronics components.

\section{Derivation of an HVAC Electric Drive Requirements}

Defining the requirements for the electric motor and its drive represents a challenging task. It starts with the analysis of the compressor type to be used: rotary compressor (RC), scroll compressor (SC) or variable displacement compressors (VDC).

In a VDC, the rotary motion of the shaft anchor plate assembly is converted to the linear motion of the piston. VDCs provide high performance at low operation speeds. From a constructive point of view, it has a small number of parts, which implies a manufacturing process with a degree of complexity and a low cost. At high speeds, however, the performance of piston compressors decreases. A SC is a rotary type compressor in which one component is in orbital motion around another that is fixed. Unlike VDC, SC have high performance at high operating speeds and a higher volumetric efficiency (the ratio of cylinder pressure at the end of the suction phase to atmospheric pressure). Due to the structure with a complex geometry, the manufacturing cost is high, and the compressor performance is reduced at low working. RCs, also known as rotary piston compressors, have either blade of vanes with the shaft. Their benefits compare to the other types of compressors are: smaller volume and weight (per unit capacity), less components, lower vibration level, higher efficiency and higher reliability. However, RCs don't offer the same cooling capacity in terms of cooled air volume and cooling time.

For FEV, HVAC systems are built mostly around a SC. Easy controllability and good efficiency at high speeds are their most important advantages for this application. Shanghai Benling Scroll Compressor company has developed several electric compressors with different sizes, with features such as: integration of the inverter inside the compressor housing and direct cooling by suction of the refrigerant. On the other hand, the Denso ES27 compressor has properties comparable to one from SANDEN and therefore can be a very good choice for cars in several categories including the entire M, N and $\mathrm{G}$ range. Information on car categories are extracted from the regulations regarding the type - approval and issuance 
of the road vehicle identity card, as well as the type - approval of the products used in them (RNTR 2).

For the present work a $4 \mathrm{~kW}, 8000 \mathrm{rpm}$ rated data for the electrical motor are chosen. In order to validate the defined characteristic, a system level evaluation is necessary. Therefore, a vehicle-level model is developed and the chosen drive is evaluated based on a well-defined driving scenario. The model is developed using LabAMESIM (Figure 1). The driving scenario was developed based on field measurements using a BMWi3.

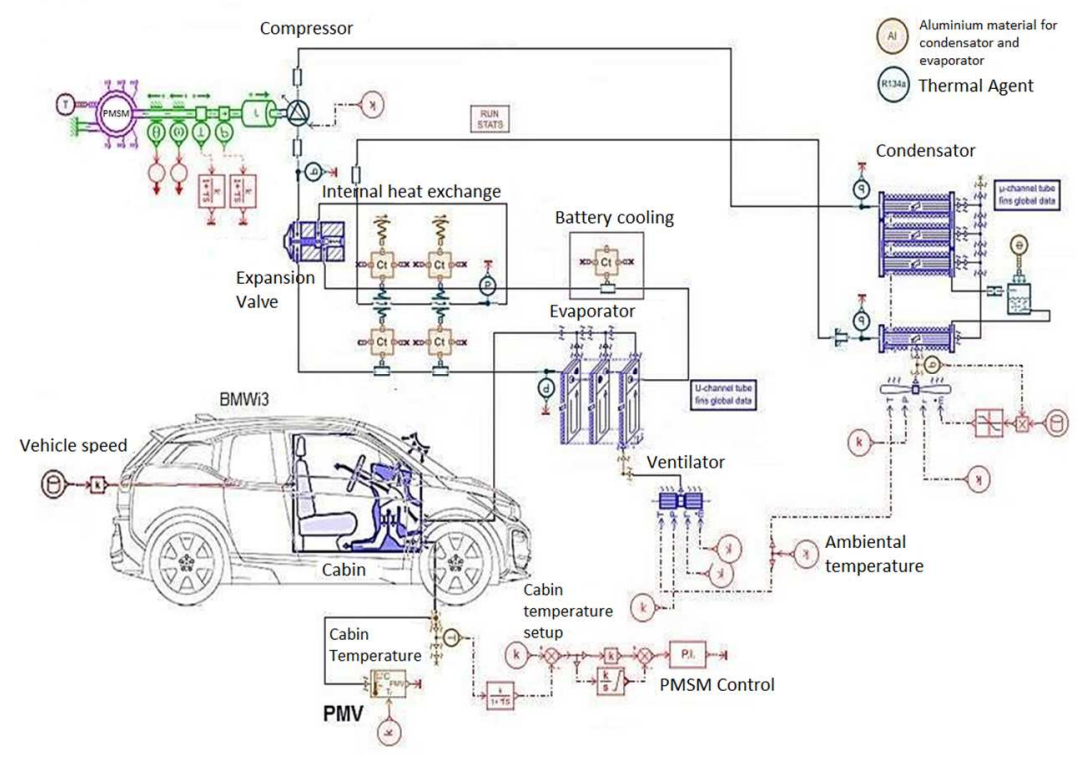

Fig. 1. System-level model for HVAC electrical motor testing.

\subsection{Development of testing scenario}

Every car manufacturer, whether with a thermal, electric or hybrid engine, tests the air conditioning system both in terms of its influence on fuel or electricity consumption in batteries and from the perspective of thermal comfort of passengers. For an electric vehicle, the ability of the system to cool the batteries must also be considered. As mentioned above, the air conditioning time in the passenger compartment is important. This cooling / heating time is directly proportional to the speed of the car, the speed of the internal fan, and depends on the external weather conditions, the performance of the compressor and the number of passengers in the car. Thermal testing of the system according to the speed of the car is done in 2 ways: (1) a dynamic regime in which the car is driven in the street traffic where we have traffic lights, pedestrian crossings, traffic signs and other vehicles. it is impossible to move the vehicle at a constant and (2) static speed where, obviously, the car is parked with the engine idling and the HVAC system running. Before designing an air conditioning system, respectively before determining the technical characteristics of the compressor, we proceeded to a reverse engineering process starting from existing systems on the market in order to have a comparison term and to validate the data transmitted by manufacturer. This requires consideration of several aspects for data acquisition, namely: (i) the temperature spectrum available in the passenger compartment, (ii) the time elapsed until the desired temperature is reached, and (iii) the HVAC performance for the static situation (when the car is parked) and dynamic (in circulation); (iv) measurement of temperature in several points: hood, ceiling, 
dashboard, ventilation hole, driver, passengers, environment. The sensors are placed on a BMWi3 at the level of ventilation hole, driver, hood, board, outdoor, cabin ceiling (See Figure 2). The measurements for the dynamic situation of the vehicle have been performed for the following conditions: the indoor temperature was set at $21^{\circ} \mathrm{C}$; fan speed has been set to the highest level; all the openings in the front panel where the cold air came from were opened; a route was chosen to allow the speed profile in Figure 3.

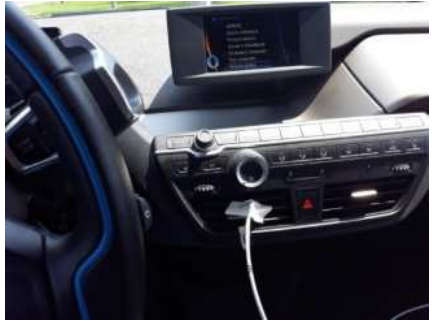

(a)

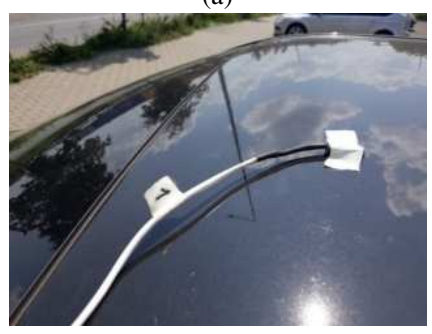

(c)

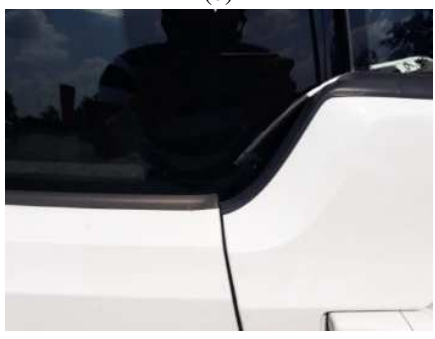

(e)

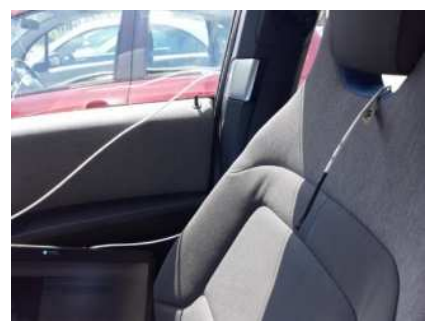

(b)

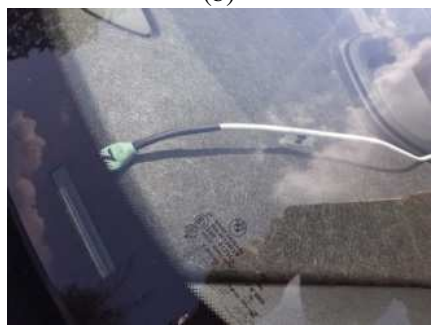

(d)

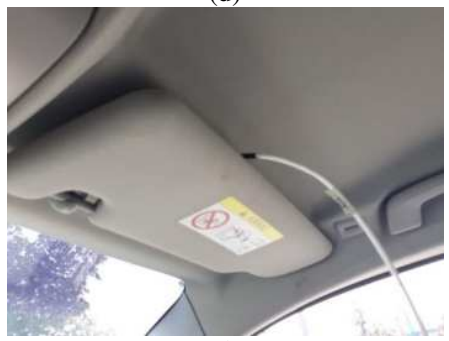

(f)

Fig. 2. Placement of sensors: (a) ventilation; (b) driver; (c) hood; (d) board; (e) outdoor; (f) ceiling.

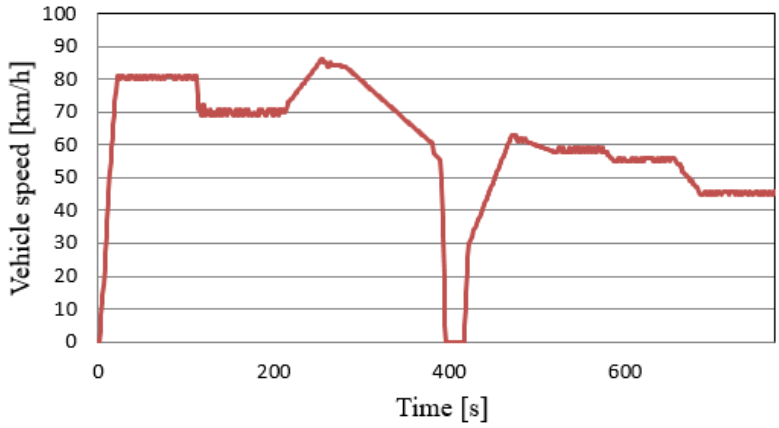

Fig. 3. Speed profile for system-level evaluation. 


\subsection{Vehicle-level evaluation and requirements derivation}

The evolution of the temperature at the level of the sensor mounted at the ventilation hole for both, on-field measurement and vehicle-level simulation are presented in Figure 4.

As conclusion, a 4kW, $8000 \mathrm{rpm}$ electrical machine answers the requirements in terms of power needed to provide a comfortable temperature in the cabin. A PMSM machine was chosen to be designed and tested for driving a SC of a FEV. Beside the rated output power and speed, other specifications are needed in order to start the design procedure. A $72 \mathrm{Vdc}$ voltage source is considered to feed the machine, and a limitation of the outer diameter and stack length of the machine is added.

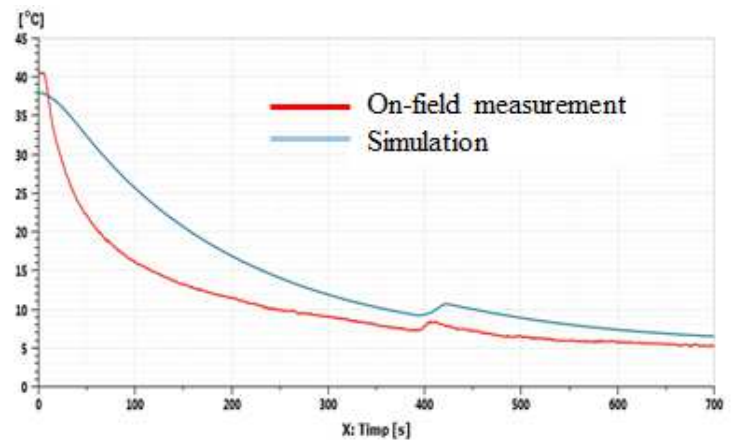

Fig. 4. Temperature evolution during the driving scenario.

\section{Design and Analysis of the PMSM for SC driving}

An 18 slot 6 pole topology (see Figure 5) was chosen for this purpose, and the geometrical data for the machine is given in Table I. The placement of the magnets is also a very important aspect, as operation at high speeds can have damaging effects on the rotor integrity. Therefore, even though the permanent magnets placement was chosen to be on the rotor surface, they are buried at a depth of $0.8 \mathrm{~mm}$, which ensures the functionality and integrity of the rotor at high speeds. The von Mises stress has been evaluated in order to verify that the PM mounting withstand the centrifugal forces generated by the operation at the rated speed.

Table 1. Main dimensions of proposed PMSM.

\begin{tabular}{|l|r|}
\hline Outer diameter [mm] & 118 \\
\hline Inner stator diameter $[\mathrm{mm}]$ & 50 \\
\hline Average tooth width $[\mathrm{mm}]$ & 2.6 \\
\hline Magnet height $[\mathrm{mm}]$ & 2 \\
\hline
\end{tabular}

The model of the machine was implemented in a FEM-based software and the results of the simulations are presented below. As it can be noticed from Figure 6, the ripple content of developed electromagnetic torque is rather high. Thus a skew factor of one slot, or $20^{\circ}$ was employed, in order to reduce this value. 


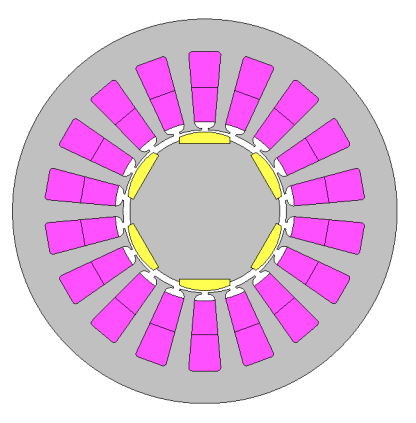

Fig. 5. PMSM topology.

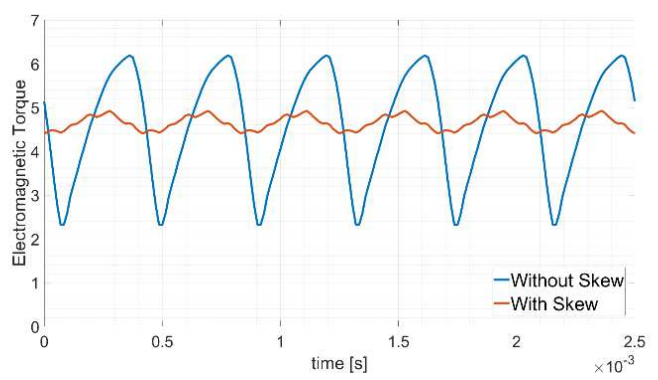

Fig. 6. Electromagnetic torque developed by the proposed topology.

The average electromagnetic torque and ripple content for the initial and skewed topologies are given in Table 2.

Table 2. Average torque and ripple content.

\begin{tabular}{|l|c|c|}
\hline & Non-Skew & Skew \\
\hline Average Torque [Nm] & 4.73 & 4.65 \\
\hline Torque Ripple [\%] & $183 \%$ & $19 \%$ \\
\hline
\end{tabular}

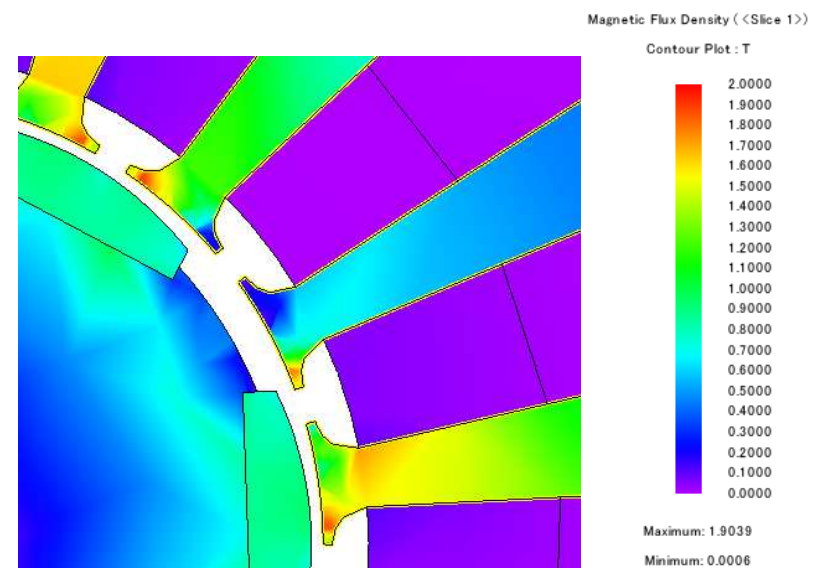

Fig. 7. Flux density map of PMSM.

Although the average torque reduces in value, this is an expected phenomenon when applying this technique, however, the torque average remains within acceptable limits. A 
magnetic flux density map can be observed in Figure 7 and ensures that the materials used are not saturated at the given power supply.

\section{HiL testing of machine}

A Hil setup was done for the PMSM, using MicroLabBox from DSpace. The load used, in order to test the performances of the PMSM, is a DC motor, and the setup can be seen in Figure 8 .

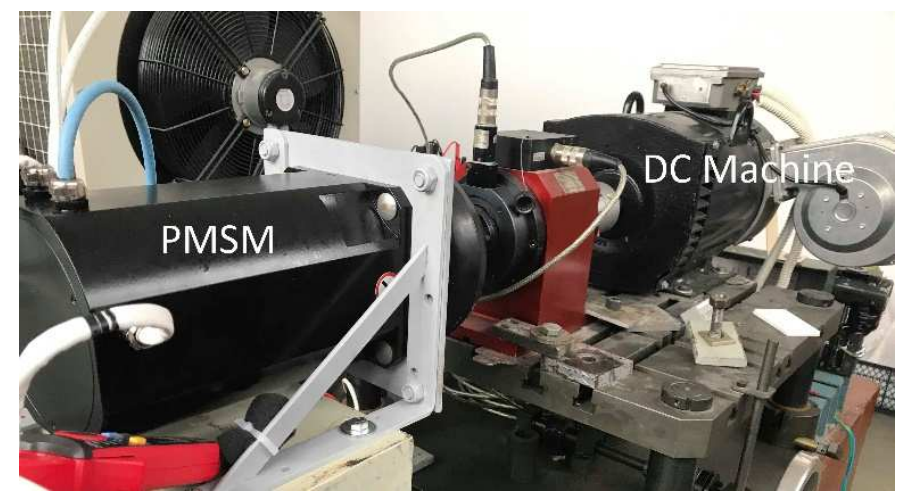

Fig. 8. Hil Setup.

The HiL setup is based on the Field Oriented Control (FOC), widely used in the control of AC machines, for being very reliable and very easy to use. The measured mechanical torque, phase voltage and phase current at different speeds are presented in Figure 10. It can be noticed that the experimental machine answer to the requirements according to which the machine was designed.
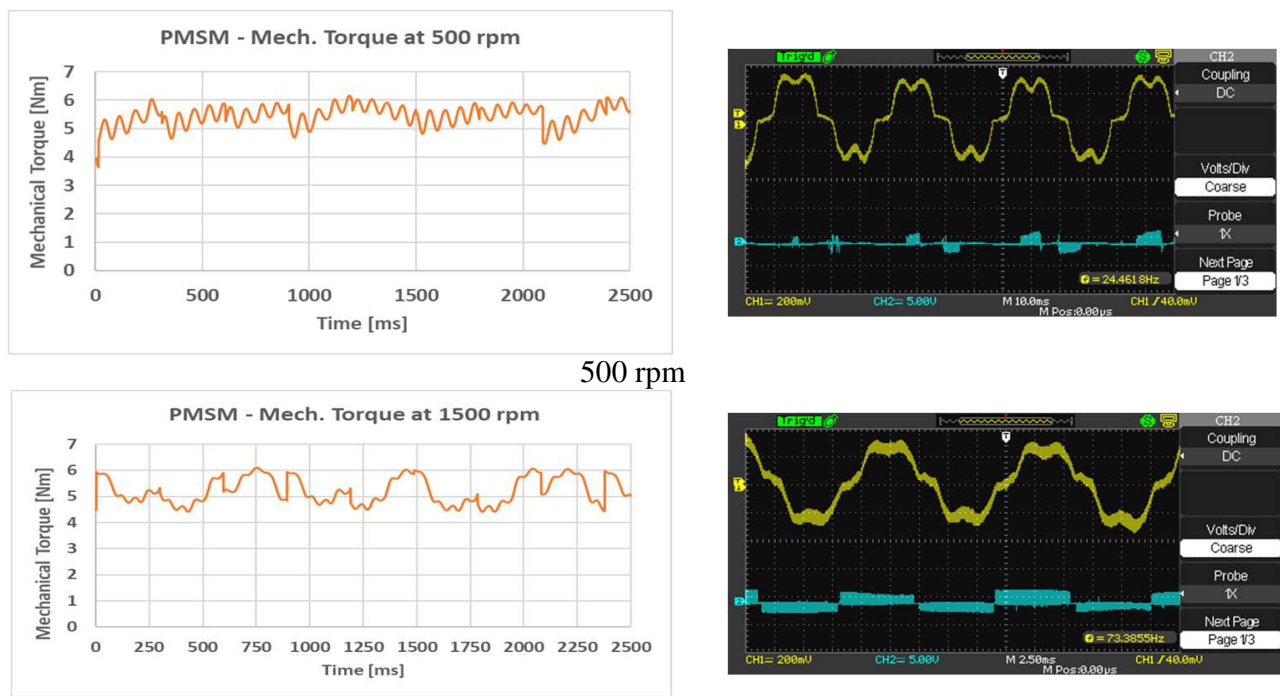

$1500 \mathrm{rpm}$

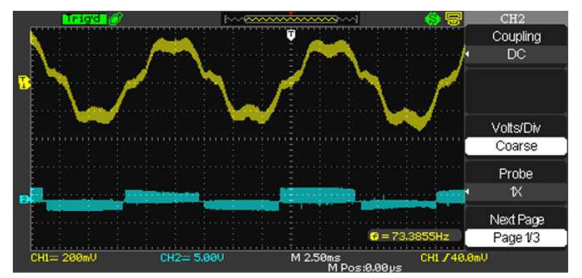




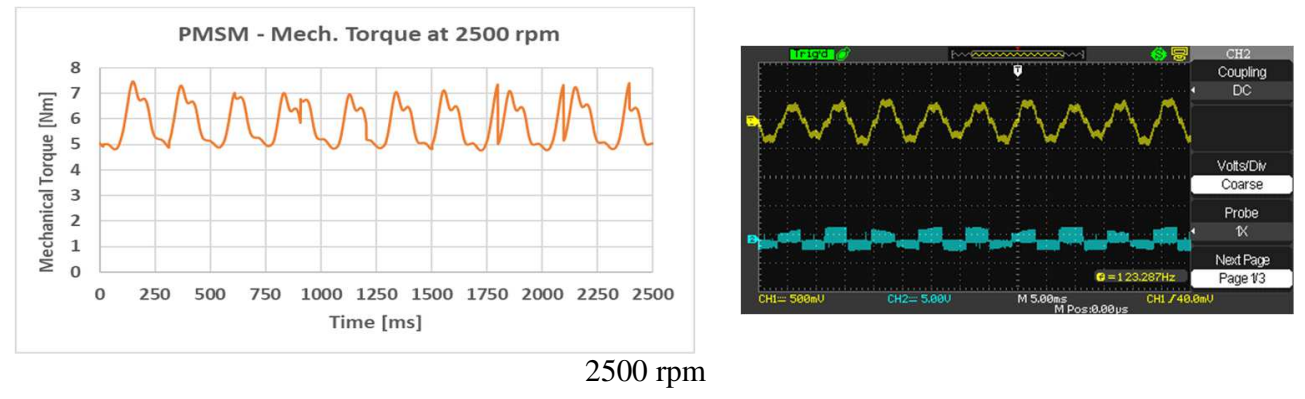

Fig. 9. Mechanical torque, phase voltage and current at different speeds.

\section{Conclusions}

PMSM is one of the most used electrical machines in electrical drives for automotive auxiliaries, including HVAC systems. The paper presented some considerations on the design, analysis and testing of this type of machines. An experimental model was developed and tested in order to validate the design. Future work is focused on the development and validation of control strategies for reducing the impact of HVAC electrical drive on the energy flow in the car.

\section{References}

1. B. Torregrosa-Jaime, J. Paya, J. M. Corberan, SAE Int. J. Altern. Powertrains (2013)

2. B. Song, J. Kwon, Y. Kim, World Electr. Veh. (2015)

3. M. Simion, L. Socaciu, P. Unguresan, Energy Procedia (2016)

4. P. Drage, M. Hinteregger, G. Zotter, M. Simek, ATZ Worldwide (2019)

5. Y. Shin, J. Ham, H. Cho, J. Mech. Sci. Technol. (2019)

6. Z. Zhang, Renew. Sust. Energ. Rev. (2018)

7. U. Bau, H. Schreiber, F. Lanzerath, A. Bardow, $26^{\text {th }}$ Aachen Colloquim Automobile and Engine Technoloy (2017)

8. M. A. Al Faruque, et.al., 21st Asia and South Pacific Design Automation Conference (2016)

9. K. Vatanparvar, M. A. Al Faruque, ACM Transactions on Embedded Computing Systems (2018)

10. S. Paulke, M. Ellinger, 2nd European Workshop on Mobile Air Conditioning and Auxiliary Systems (2007)

11. R. K. Shah, 3rd BSME-ASME International Conference on Thermal Engineering (2006)

12. https://www.edn.com/fundamentals-of-the-automotive-cabin-climate-control-system/

13. M. Jeffers, L. Chaney, J. Rugh, SAE 2016 World Cingress and Exhibition (2016)

14. L. Tang, G.J. Su, IEEE Power Electronics Specialists Conference (2007)

15. M. Naidu, M. T. W. Nehl, S. Gopalakrishnan, L. Wurth, IEEE Ind. Appl. Mag. 11(4) (2005) 